\title{
Comunicação
}

\section{A aplicação foliar de molibdênio na fase de enchimento de vagens do feijão-comum pode reduzir a qualidade da semente}

\author{
Rogério Faria Vieira*1, Trazilbo José de Paula Júnior ${ }^{1}$, Adalgisa Leles do Prado², Roberto Fontes Araújo ${ }^{1}$, \\ Miller da Silva Lehner ${ }^{3}$, Rhaphael Alves Silva \\ http://dx.doi.org/10.1590/0034-737X201562040012
}

\section{RESUMO}

Com o objetivo de aprimorar a tecnologia de produção de sementes ricas em molibdênio (Mo), foram avaliados os efeitos da aplicação foliar de $600 \mathrm{~g} \mathrm{ha}^{-1}$ de Mo na qualidade fisiológica da semente de feijão. Em campo, essa dose de Mo foi aplicada na fase V4 (terceira folha trifoliolada), ou parcelada de duas a quatro vezes e aplicada em V4, R6 (floração), R7 e, ou, R8 (enchimento de vagens). A aplicação de $300 \mathrm{~g} \mathrm{ha}^{-1}$ de Mo em R6 não influenciou a qualidade da semente, em comparação com a do controle ( $90 \mathrm{~g} \mathrm{ha}^{-1}$ de Mo aplicado em V4). Dos três tratamentos em que 200, 255 ou $300 \mathrm{~g} \mathrm{ha}^{-1}$ de Mo foram aplicados em R7, apenas com $255 \mathrm{~g} \mathrm{ha}^{-1}$ o Mo reduziu a germinação e vigor das sementes, em comparação com as do controle. Quando 200, $255 \mathrm{ou} 300 \mathrm{~g} \mathrm{ha}{ }^{1}$ de Mo foram aplicados em R8 (cinco tratamentos), geralmente o Mo reduziu a germinação e, em dois casos, também o vigor das sementes, em comparação com os do controle. Concluiu-se que $255 \mathrm{~g} \mathrm{ha}^{-1}$ de Mo aplicado em R7 e, sobretudo, doses iguais ou mais altas que $200 \mathrm{~g} \mathrm{ha}^{-1}$ de Mo aplicadas em R8 podem reduzir a qualidade fisiológica da semente de feijão.

Palavras-chave: Phaseolus vulgaris, molibdênio na semente, germinação, vigor de semente.

\section{ABSTRACT}

\section{Foliar molybdenum applied to common bean pod filling may reduce seed quality}

Aiming to improve the technology of producing seed with high Mo content, the effects of $600 \mathrm{~g} \mathrm{ha}^{-1}$ of foliar-applied Mo on common bean seed quality were evaluated. In the field, this Mo rate was applied at the V4 growth stage (third trifoliolate leave) or divided into two or four rates applied at V4, R6 (flowering), R7 and/or R8 (pod filling). Mo (300 $\left.\mathrm{g} \mathrm{ha}^{-1}\right)$ applied at R6 did not affect seed quality relative to control $\left(90 \mathrm{~g} \mathrm{ha}^{-1}\right.$ of Mo applied at V4). In the three treatments in which 200, 255 or $300 \mathrm{~g} \mathrm{ha}^{-1}$ of Mo were applied at R7, only in one $\left(255 \mathrm{~g} \mathrm{ha}^{-1}\right) \mathrm{Mo}$ decreased seed germination and vigor relative to control. When 200, 255 or $300 \mathrm{~g} \mathrm{ha}^{-1}$ of Mo were applied at R8 (five treatments), in general, Mo decreased seed germination and, in two cases, also seed vigor relative to control. It was concluded that $255 \mathrm{~g} \mathrm{ha}^{-1}$ of Mo applied at R7 and, especially, doses of $200 \mathrm{~g} \mathrm{ha}^{-1}$ of Mo or higher applied at $\mathrm{R} 8$ can reduce common bean seed quality.

Key words: Phaseolus vulgaris, molybdenum in seed, germination, seed vigor.

\footnotetext{
Submetido em 23/04/2014 e aprovado em 24/06/2015.

'Epamig,Viçosa, Minas Gerais, Brasil.rfvieira@epamig.br; trazilbo@epamig.br; roberto.araujo@epamig.br

${ }^{2}$ Universidade Federal de Viçosa, Viçosa, Minas Gerais, Brasil. adalgisa.prado@ufv.br

${ }^{3}$ Universidade Federal de Viçosa, Viçosa, Minas Gerais, Brasil. miller.lehner@ufv.br

${ }^{4}$ Universidade Federal de Viçosa, Viçosa, Minas Gerais, Brasil. rhaphael.silva @ufv.br

*Autor para correspondência: rfvieira@epamig.br
} 


\section{INTRODUÇÃO}

O molibdênio (Mo) é exigido em pequena quantidade pela planta e, nela, suas funções estão associadas a mudanças de valência, ao participar de enzimas. Oxidado, ele existe como Mo (VI), reduzido, como Mo (V) e Mo (IV). O Mo participa das enzimas nitrogenase e nitrato redutase. Esta última é chave na assimilação do nitrato. Logo, quando há deficiência dessa enzima, a planta depende do amônio como fonte de nitrogênio $(\mathrm{N})$. A nitrogenase é necessária para a fixação biológica do $\mathrm{N}$ (Mendel, 2011).

Em feijão-comum (Phaseolus vulgaris), a prevenção ou correção da deficiência de Mo geralmente é feita pelo tratamento de sementes ou, sobretudo, pela pulverização de plantas com solução de Mo. Esses métodos são usados por agricultores bem informados e em regiões onde há disponibilidade de adubo molíbdico no comércio. Recentemente, com o aprimoramento da técnica para enriquecer sementes com Mo (Vieira et al., 2005, Vieira et al., 2011; Pacheco et al., 2012), é possível fornecer aos agricultores, a baixo custo (Vieira et al., 2011), esse micronutriente, por meio da semente. Nesse caso, a disponibilização de Mo para a planta independe de a tecnologia ser conhecida, ou não, pelo agricultor, e de o adubo molíbdico estar disponível, ou não, no comércio local. Para produzir sementes ricas em Mo, as plantas são pulverizadas, geralmente mais de uma vez, com doses de Mo mais altas que as usadas para prevenir ou corrigir deficiência de Mo no solo.

Na Zona da Mata de Minas Gerais, para se prevenir ou corrigir deficiência de Mo do solo, aplicam-se de 70 a $100 \mathrm{~g} \mathrm{ha}^{-1} \mathrm{de}$ Mo, entre 14 e 28 dias após a emergência dos feijoeiros (Berger et al., 1996). Sementes colhidas de feijoeiros pulverizados com doses nessa faixa e nessa época de aplicação têm melhor qualidade fisiológica que as colhidas de plantas que não receberam Mo (Meireles et al., 2003; Leite et al., 2009).

Para produzir sementes ricas em Mo, a dose a ser usada depende da disponibilidade de Mo no solo, $\mathrm{pH}$ do solo, do cultivar, etc. Na Zona da Mata, $600 \mathrm{~g} \mathrm{ha}^{-1}$ de Mo geralmente elevam o conteúdo de Mo para pelo menos 3,6 $\mu \mathrm{g}$ por semente (Vieira et al., 2014), teor com que os feijoeiros não mais respondem à aplicação de Mo na folhagem (Vieira et al., 2011). Nessa região, o conteúdo de Mo da semente de feijão quase dobrou quando a dose de $1440 \mathrm{~g} \mathrm{ha}^{-1}$ de Mo foi dividida em quatro subdoses e aplicada entre V4 (terceira folha trifoliolada) e R5 (pré-floração), em comparação com o efeito da aplicação única em V4 (Vieira et al. 2005). Há indicações de que a aplicação do Mo na fase reprodutiva do feijão (Kubota et al., 2008) seja boa estratégia para produzir sementes ricas nesse nutriente. Em soja, $1000 \mathrm{~g}$ ha $^{-1}$ de Mo aplicados durante a fase de enchimento de vagens não influenciou a qualidade fisiológica das sementes (Milani et al., 2010). No entanto, em relação ao feijão-comum, não há estudo a esse respeito. $\mathrm{O}$ objetivo deste trabalho foi avaliar os efeitos de parcelamentos e de épocas de aplicação do adubo molíbdico em fases reprodutivas do feijão-comum sobre a qualidade fisiológica das sementes colhidas.

\section{MATERIAL E MÉTODOS}

Instalou-se ensaio, em 14 de março de 2010, em Coimbra ( $20^{\circ} 49^{\prime} \mathrm{S}, 42^{\circ} 45^{\prime} \mathrm{W}$, altitude de $716 \mathrm{~m}$ ), município da Zona da Mata de Minas Gerais, em Argissolo Vermelho-Amarelo com $560 \mathrm{~g} \mathrm{~kg}{ }^{1}$ de argila, $190 \mathrm{~g} \mathrm{~kg}{ }^{1}$ de silte e $250 \mathrm{~g} \mathrm{~kg}^{1}$ de areia e deficiente em Mo (Vieira et al., 2005). Na instalação do ensaio, a análise do solo na camada de $0-20 \mathrm{~cm}$ indicou: $\mathrm{pH}$ em água (relação 1:2,5) de 4,8; 11 e $96 \mathrm{mg} \mathrm{dm}^{-3}$ de $\mathrm{P}$ e $\mathrm{K}$ disponíveis, respectivamente (extrator Mehlich); 0,5, 1,7 e 0,8 $\mathrm{cmol}_{\mathrm{c}}$ $\mathrm{dm}^{-3} \mathrm{de} \mathrm{Al}, \mathrm{Ca}$ e $\mathrm{Mg}$, respectivamente, extraídos com $\mathrm{KCl}$ mol L ${ }^{-1} ; 34 \%$ de saturação por bases e $1,4 \mathrm{dag} \mathrm{kg}^{-1}$ de matéria orgânica (Walkley-Black). O solo foi preparado com grade aradora, seguida de grade niveladora.

Os tratamentos constaram de formas de parcelamento de $600 \mathrm{~g} \mathrm{ha}^{-1}$ de Mo, nestas fases: V4, R6 (floração), R7 (formação de vagens) e,ou, R8 (enchimento de vagens) (Tabela 1). Aplicada em V4, a dose de $90 \mathrm{~g} \mathrm{ha}^{-1}$ de Mo, recomendada para prevenir ou corrigir deficiência de Mo na Zona da Mata (Berger et al., 1996), foi usada como controle.

Usou-se o cultivar BRSMG Majestoso, do grupo comercial carioca e de hábito de crescimento indeterminado (tipo III), que é bom acumulador de Mo na semente (Vieira et al., 2014). No sulco de plantio, distribuíram-se $350 \mathrm{~kg} \mathrm{ha}^{-1}$ do formulado 8-28-16 $\left(\mathrm{N}-\mathrm{P}_{2} \mathrm{O}_{5}\right.$ $-\mathrm{K}_{2} \mathrm{O}$ ). Em V4, os feijoeiros de todas as parcelas receberam, além das respectivas adubações com Mo (Tabela 1), adubação em cobertura, com $100 \mathrm{~kg} \mathrm{ha}^{-1}$ de ureia, distribuídos em filete e a $10 \mathrm{~cm}$ da fileira. Com pulverizador de $\mathrm{CO}_{2}$ pressão de $245 \mathrm{kPa}$, equipado com bicos XR 11002 espaçados de $0,5 \mathrm{~m}$, distribuiu-se o molibdato de sódio $\left(\mathrm{Na}_{2} \mathrm{MoO}_{4} 2 \mathrm{H}_{2} \mathrm{O}\right)$ sobre a folhagem. Adicionouse à solução de Mo um espalhante adesivo. Lona plástica foi estendida entre as parcelas vizinhas às tratadas para interceptar a deriva. Fez-se o controle de plantas daninhas com os herbicidas fomesafen $\left(0,25 \mathrm{~kg} \mathrm{ha}^{-1}\right) \mathrm{e}$ fluazifop-p-butil $\left(0,20 \mathrm{~kg} \mathrm{ha}^{-1}\right) \mathrm{e}$, quando necessário, também com capinas manuais. Controlaram-se pragas com os inseticidas metamidofós $\left(500 \mathrm{~mL} \mathrm{ha}^{-1}\right)$ ou clorpirifós $\left(400 \mathrm{~mL} \mathrm{ha}^{-1}\right)$ e doenças foliares com o fungicida epoxiconazol $\left(12,5 \mathrm{~mL} \mathrm{ha}^{-1}\right)$ ou azoxystrobin $\left(50 \mathrm{~g} \mathrm{ha}^{-1}\right)$. O fungicida fluazinam $\left(0,5 \mathrm{~L} \mathrm{ha}^{-1}\right)$ foi aplicado no início 
da floração e dez dias depois, para o controle preventivo do mofo-branco (Sclerotinia sclerotiorum). Irrigações semanais foram feitas com aproximadamente 40 (fase vegetativa) ou $50 \mathrm{~mm}$ (fase reprodutiva) de água.

Foi usado o delineamento em blocos ao acaso, com sete repetições. As parcelas constaram de quatro fileiras de $2 \mathrm{~m}$, espaçadas de 0,6 m. Foram distribuídas 15 sementes por metro. A parcela útil constou das duas fileiras centrais.

Avaliaram-se estande final, produtividade, massa de 100 sementes, germinação e vigor das sementes colhidas. A produtividade e a massa de 100 sementes foram obtidas com sementes com aproximadamente $13 \%$ de água (base úmida). Utilizaram-se os seguintes testes de vigor: envelhecimento acelerado, condutividade elétrica e frio. Nos testes de germinação e de vigor, realizados 35 dias após a colheita, usaram-se quatro replicatas de 50 sementes de cada tratamento de campo. Os testes de germinação, envelhecimento acelerado e condutividade elétrica foram realizados conforme descrito por Leite et al. (2009). No teste de frio, as sementes foram distribuídas em papel germitest previamente umedecido, como feito no teste de germinação. Os rolos foram colocados no interior de sacos plásticos, vedados com fita adesiva e mantidos em câmara incubadora BOD, a $10^{\circ} \mathrm{C}$, durante sete dias (Loeffler et al., 1985). Em seguida, os rolos foram transferidos para germinador, regulado à temperatura de $25^{\circ} \mathrm{C}$, onde permaneceram por cinco dias antes da contagem de plântulas normais (Brasil,
2009). Nos testes de germinação e vigor, usou-se o delineamento inteiramente casualizado, com sete repetições.

Avaliaram-se, ademais, o teor de molibdênio das sementes provenientes de três tratamentos: 90 (V4), 600 (V4) e 90 (V4) + 170 (R6) + 170 (R7) + 170 (R8). Fezse essa avaliação com as sete repetições de campo, com o objetivo de confirmar se o Mo aplicado na folhagem aumentou o teor de Mo da semente. A metodologia usada para avaliar o teor de Mo das sementes é descrita por Vieira et al. (2005).

Os dados, previamente transformados por arco seno de $\sqrt{x / 100}$, foram submetidos à análise de variância. Utilizou-se o teste de Dunnett para comparar os tratamentos de parcelamento de $600 \mathrm{~g} \mathrm{ha}^{-1}$ de Mo com o controle (90 $\mathrm{g} \mathrm{ha}^{-1}$ de Mo aplicado em V4).

\section{RESULTADOS E DISCUSSÃO}

Os tratamentos não influenciaram o estande final (F $=0,778, p=0,660, \mathrm{CV}=21,8 \%$, média de 177 mil plantas $\left.\mathrm{ha}^{-1}\right)$, a produtividade de grãos $(\mathrm{F}=0,804, p=0,636$, $\mathrm{CV}=10,5 \%$, média $\left.=4148 \mathrm{~kg} \mathrm{ha}^{-1}\right)$ e a massa de 100 sementes $(\mathrm{F}=0,440, p=0,949, \mathrm{CV}=6,1 \%$, média $=$ $27,3 \mathrm{~g})$. $\mathrm{O}$ efeito não significativo dos tratamentos na produtividade era esperado, pois os feijoeiros de todos os tratamentos receberam Mo e $\mathrm{N}$ em cobertura.

As sementes originadas de plantas adubadas com 90, 600 (ambas em V4) e $600 \mathrm{~g} \mathrm{ha}^{-1}$ de Mo parcelados em V4, R6, R7 e R8, apresentaram teor médio de Mo de 0,$59 ; 11,83$ e $15,2 \mu \mathrm{g} \mathrm{g}^{-1}$, respectivamente. Portanto, os

Tabela 1. Percentagem de germinação de sementes (PGS), PGS após envelhecimento acelerado (EA) e estresse de frio (EF) em função de parcelamentos de $600 \mathrm{~g} \mathrm{ha}^{-1}$ de Mo em até quatro fases de desenvolvimento do feijão, em Coimbra, MG

\begin{tabular}{|c|c|c|c|}
\hline $\begin{array}{l}\text { Dose de molibdênio em } \mathrm{g} \mathrm{ha}^{-1} \\
\text { (fase de desenvolvimento do feijão) }^{(1)}\end{array}$ & $\operatorname{PGS}^{(2)}(\%)$ & $\mathbf{E A}^{(2)}(\%)$ & $\mathbf{E F}^{(2)}(\%)$ \\
\hline $90(\mathrm{~V} 4)$ & 81,8 & 86,4 & 92,7 \\
\hline $600(\mathrm{~V} 4)$ & 79,4 & 83,3 & 91,1 \\
\hline $300(\mathrm{~V} 4)+300(\mathrm{R} 6)$ & 77,7 & 83,1 & 88,9 \\
\hline $300(\mathrm{~V} 4)+300(\mathrm{R} 7)$ & 76,7 & 85,8 & 89,3 \\
\hline $300(\mathrm{~V} 4)+300(\mathrm{R} 8)$ & $72,1 *$ & $77,3^{*}$ & $83,1^{*}$ \\
\hline $90(\mathrm{~V} 4)+255(\mathrm{R} 6)+255(\mathrm{R} 7)$ & $74,0^{*}$ & $78,7 *$ & 87,4 \\
\hline $90(\mathrm{~V} 4)+255(\mathrm{R} 6)+255(\mathrm{R} 8)$ & $72,4^{*}$ & $79,0^{*}$ & 86,7 \\
\hline $90(\mathrm{~V} 4)+255(\mathrm{R} 7)+255(\mathrm{R} 8)$ & $75,3^{*}$ & 85,0 & 89,7 \\
\hline $200(\mathrm{~V} 4)+200(\mathrm{R} 6)+200(\mathrm{R} 7)$ & 76,9 & 83,1 & 91,1 \\
\hline $200(\mathrm{~V} 4)+200(\mathrm{R} 6)+200(\mathrm{R} 8)$ & $73,8^{*}$ & 81,3 & 89,0 \\
\hline $200(\mathrm{~V} 4)+200(\mathrm{R} 7)+200(\mathrm{R} 8)$ & 76,9 & 83,6 & 88,7 \\
\hline $90(\mathrm{~V} 4)+170(\mathrm{R} 6)+170(\mathrm{R} 7)+170(\mathrm{R} 8)$ & 77,2 & 86,8 & 90,9 \\
\hline Média & 76,2 & 82,8 & 89,1 \\
\hline $\mathrm{CV}(\%)$ & 5,1 & 5,7 & 5,0 \\
\hline
\end{tabular}


feijoeiros que receberam $600 \mathrm{~g} \mathrm{ha}^{-1}$ de Mo chegaram a produzir sementes com teor de Mo 26 vezes mais alto que os que receberam $90 \mathrm{~g} \mathrm{ha}^{-1}$ de Mo. O teor de Mo mais alto neste estudo ficou um pouco abaixo do obtido em Coimbra, com o cultivar Majestoso, no estudo de Vieira et al. (2014).

$\mathrm{O}$ teste de condutividade elétrica não detectou diferença significativa entre tratamentos $(\mathrm{F}=0,686, p=$ $0,747, \mathrm{CV}=10,3 \%$ ). A dose de $600 \mathrm{~g} \mathrm{ha}^{-1}$ de Mo aplicados em V4 não influenciou significativamente a germinação e o vigor das sementes, em comparação com os do controle, com $90 \mathrm{~g} \mathrm{ha}^{-1}$ de Mo (Tabela 1). A aplicação de $300 \mathrm{~g} \mathrm{ha}^{-1}$ de Mo em V4 e, em seguida, de $300 \mathrm{~g} \mathrm{ha}^{-1}$ em R6 ou R7 também não influenciou a germinação e o vigor das sementes, mas a diferença entre as médias das variáveis dependentes do tratamento $300(\mathrm{~V} 4)+300$ (R8), em comparação com os do controle, foi significativa.

Os três tratamentos com aplicação de $255 \mathrm{~g} \mathrm{ha}^{-1}$ de Mo em R7 ou R8 (após aplicação de $90 \mathrm{~g} \mathrm{ha}^{-1}$ de Mo em V4 e $255 \mathrm{~g} \mathrm{ha}^{-1}$ em R6 ou R7), reduziram a germinação e, em dois casos, o vigor das sementes (teste de envelhecimento acelerado). Um dos três tratamentos com aplicação de $200 \mathrm{~g} \mathrm{ha}^{-1}$ de Mo em R7 ou R8 (após $200 \mathrm{~g}$ ha $^{-1}$ em V4 e $200 \mathrm{~g} \mathrm{ha}^{-1}$ em R6 ou R7), causou redução da germinação, em comparação com a do controle. Os resultados desses seis tratamentos, em que a dose de Mo foi parcelada três vezes, podem estar indicando que o aumento da dose usada em V4, de 90 para $200 \mathrm{~g} \mathrm{ha}^{-1}$ de Mo, com consequente redução das doses usadas na fase reprodutiva do feijão, pode minimizar os efeitos danosos do Mo na qualidade das sementes. Desconhece-se o mecanismo que explica os efeitos de doses relativamente altas de Mo, aplicadas na fase reprodutiva do feijão, na qualidade fisiológica das sementes colhidas.

Considerados em conjunto, os resultados do parcelamento da dose de Mo em duas ou três aplicações (nove tratamentos) sugerem que 200 e, sobretudo, 255 ou 300 $\mathrm{g} \mathrm{ha}^{-1}$ de Mo aplicados em R8, podem reduzir a qualidade fisiológica das sementes. Também indica que $255 \mathrm{~g}$ $\mathrm{ha}^{-1}$ de Mo aplicados em R7 (depois de $255 \mathrm{~g} \mathrm{ha}^{-1} \mathrm{em}$ R6) podem reduzir a qualidade fisiológica das sementes. Em outro estudo, conduzido na Zona da Mata, a aplicação de $1000 \mathrm{~g} \mathrm{ha}^{-1}$ de Mo, aos 71 DAE (fase R7), não influenciou significativamente a germinação das sementes (Vieira et al., 2010). Logo, mais estudos com combinações de doses de Mo aplicadas em R7 são necessários para esclarecer essa questão. Em soja adubada com 400 a $1000 \mathrm{~g} \mathrm{ha}^{-1}$ de Mo (uma ou duas aplicações) na fase de enchimento de vagens não mostraram efeito significativo dos tratamentos na qualidade fisiológica da semente (Milani et al., 2010). No entanto, além de esses autores trabalharem com sementes de espécie com características diferentes (especialmente quanto a óleo e proteína) das do feijão-comum, eles não usaram controle com dose baixa de Mo para comparação e, também, não empregaram o teste de envelhecimento acelerado. Este teste de vigor foi o que mais diferenciou os tratamentos, neste estudo.

A diferença não significativa entre as médias de germinação e de vigor do tratamento 90 (V4) + 170 (R6) + $170(\mathrm{R} 7)+170(\mathrm{R} 8)$ e as do controle sugerem que o uso de dose de $170 \mathrm{~g} \mathrm{ha}^{-1}$ de Mo, ou mais baixas, mesmo se aplicadas em R8, não reduzem a qualidade fisiológica das sementes.

\section{CONCLUSÕES}

Dose de $300 \mathrm{~g} \mathrm{ha}^{-1}$ de Mo aplicada na floração (R6) do feijão-comum não influencia a qualidade fisiológica das sementes.

Dose de $255 \mathrm{~g} \mathrm{ha}^{-1}$ de Mo aplicada na fase de formação de vagens (R7), antecedida da aplicação de $255 \mathrm{~g} \mathrm{ha}^{-1}$ de Mo em R6, pode reduzir a qualidade fisiológica das sementes.

Dose de $200 \mathrm{~g} \mathrm{ha}^{-1}$ de Mo e, sobretudo, doses mais altas que essa, aplicadas na fase de enchimento de vagens (R8) podem reduzir a qualidade das sementes, mas dose de $170 \mathrm{~g} \mathrm{ha}^{-1}$ de Mo, mesmo aplicada em R8, não influencia a qualidade fisiológica das sementes.

\section{AGRADECIMENTOS}

À Fapemig, pelo financiamento deste estudo; ao CNPq, belas bolsas concedidas.

\section{REFERÊNCIAS}

Berger PG, Vieira C \& Araújo GAA (1996) Efeitos de doses e épocas de aplicação de molibdênio sobre a cultura do feijão. Pesquisa Agropecuária Brasileira, 31:473-480.

Brasil (2009) Regras para análise de sementes. Brasília, Mapa/ACS. 399p.

Kubota FY, Andrade Neto AC de, Araújo AP \& Teixeira MG (2008) Crescimento e acumulação de nitrogênio de plantas de feijoeiro originadas de sementes com alto teor de molibdênio. Revista Brasileira de Ciência do Solo, 32:1635-1641.

Leite UT, Araújo GAA, Miranda GV, Vieira RF \& Pires AA (2009) Influência do conteúdo de molibdênio na qualidade fisiológica da semente de feijão: cultivares Novo Jalo e Meia Noite. Revista Ceres, 56:225231.

Loeffler TM, Meyer JL \& Burris JS (1985) Comparision of two test procedures for use in maize drying studies. Seed Science and Technology, 13:653-658.

Meireles RC, Reis LS dos, Araújo EF, Soares A da S \& Araújo GAA (2003) Efeito da época e do parcelamento de aplicação de molibdênio, via foliar, na qualidade fisiológica das sementes de feijão. Revista Ceres, 50:699-707.

Mendel RR (2011) Cell biology of molybdenum in plants. Plant Cell Report, 30:1787-1797. 
Milani GL, Oliveira JA, Pereira E de M, Carvalho BO, Oliveira GE \& Costa RR (2010) Aplicação foliar de molibdênio durante a maturação de sementes de soja. Ciência e Agrotecnologia, 34:810-816.

Pacheco RS, Brito LF, Staliotto R, Pérez DV \& Araújo AP(2012) Seeds enriched with phosphorus and molybdenum as a strategy for improving grain yield of common bean crop. Field Crops Research, 136:97-106

Vieira RF, Salgado LT \& Ferreira ACC (2005) Performance of common bean using seeds harvested from plants fertilized with high rates of molybdenum. Journal of Plant Nutrition, 28:393-377.
Vieira RF, Salgado LT, Pires AA \& Rocha GS (2010) Conteúdo de molibdênio das sementes de feijoeiro em resposta a doses do micronutriente pulverizado sobre as plantas. Ciência Rural, 40:666669.

Vieira RF, Paula Júnior TJ, Pires AA, Carneiro JES \& Rocha GS (2011) Common bean seed complements molybdenum uptake by plants from soil. Agronomy Journal, 103:1843-1848.

Vieira RF, Paula Júnior TJ, Carneiro JES \& Queiroz MV (2014) Genotypic variability in seed accumulation of foliar-applied molybdenum to common bean. Revista Brasileira de Ciência do Solo, 38:205-213. 